\title{
Acondicionamiento de un Túnel de Viento para pruebas aerodinámicas y prácticas de laboratorio en la UNAH
}

\author{
Omri Alberto Amaya Carias \\ Ivan Vladimir Betancourt Mendoza
}

\section{Resumen}

La finalidad de este artículo es examinar las condiciones en que se encuentra el túnel de viento existente en el Laboratorio de Máquinas Herramientas de la Facultad de Ingeniería de la UNAH. Se presenta un inventario detallado de las partes existentes y se propone la lista de las partes faltantes y la cotización de cada una de ellas. El túnel habilitado permitirá examinar a escala la interacción entre las superficies alares propias de las aeronaves modernas y el flujo de aire. La metodología utilizada se desarrolló en tres momentos: primero se hizo una revisión bibliográfica para definir los conceptos básicos de aerodinámica aplicables al vuelo de aeronaves y susceptibles de ser demostrados en un laboratorio; en un segundo momento, se recolectó información exhaustiva sobre las condiciones en las que se encuentra actualmente el túnel existente en UNAH; en un tercer momento, se establecieron los productos y conclusiones de la investigación. Se concluye que un túnel de viento activado, constituiría un recurso pedagógico importante para explicar principios teóricos de aerodinámica de muchas de las asignaturas impartidas en la UNAH; además, se considera factible la adecuación del artefacto existente o la compra de uno nuevo; en el segundo caso se recomienda que se adquiera un túnel automatizado. El presente trabajo inaugura una nueva línea de investigación aeronáutica en Honduras, para cuya profundización queda pendiente el diseño de un manual de prácticas de laboratorio que guíe el uso del Túnel de Viento de la UNAH una vez que este se encuentre funcionando.

Palabras clave: educación aeronáutica, ciencias aeronáuticas, túnel de viento, aerodinámica, laboratorio. 


\section{Abstract}

The purpose of this article is to examine the conditions in which is the existing wind tunnel at the Laboratory for Machine Tools of the Faculty of Engineering of the UNAH. A detailed inventory of existing parts is provided and the list of the missing parts and the price of each one of them is proposed. The tunnel enabled allow scale examine the interaction between alar surfaces of modern aircraft and air flow. The methodology was developed in three phases: first a Bibliographic review to define the basic concepts of aerodynamics applicable to the flight of aircraft and capable of being demonstrated in a laboratory was made; in a second phase, comprehensive information on the conditions under which the existing tunnel currently in UNAH was collected; in a third phase, products and conclusions were established. It is concluded that an activated wind tunnel would be an important educational resource to explain theoretical principles of aerodynamics of many of the courses taught at the UNAH; moreover, the adequacy of the existing device or buying a new one is considered feasible; in the second case it is recommended that an automated tunnel is acquired. This work opens a new line of aeronautics research in Honduras, whose deepening is pending for the design of a manual laboratory practice to guide the use of Wind Tunnel UNAH, once it is operating.

Keywords: aviation education, aeronautical science, wind tunnel, aerodynamics, laboratory.

Omri Alberto Amaya Carias (omri.amaya@unah.edu.hn),Departamento de Ciencias Aeronáuticas, Facultad de Ciencias Espaciales, Universidad Nacional Autónoma de Honduras. Ivan Vladimir Betancourt Mendoza ( ivan.betancourt@ unah.edu.hn),Departamento de Ciencias Aeronáuticas,Facultad de Ciencias Espaciales,Universidad Nacional Autónoma de Honduras. 


\section{INTRODUCCIÓN}

Los túneles aerodinámicos, también llamados túneles de viento, dada su traducción literal de la expresión inglesa "wind tunnel", es un sistema que genera una corriente de aire de manera que pueda ser utilizado como instrumento científico tecnológico, cuya aplicación se basa en lograr condiciones especiales en el flujo y simular el movimiento de objetos con el fin de analizar fenómenos aerodinámicos, como fuerzas y líneas de corriente, entre otros. En el túnel también es posible analizar el rendimiento y funcionamiento de ventiladores generando sus curvas características.

El presente artículo se basa en un túnel de viento ya existente en el Laboratorio de Máquinas Herramientas, de la Facultad de Ingeniería de la Universidad Nacional Autónoma de Honduras, el que en su estado actual permite un reacondicionamiento para hacerlo operativo, lo que podría convertirlo en el único túnel de viento funcional para procesos de investigación y enseñanza en Honduras.

El reacondicionamiento del túnel de viento de la UNAH para realizar pruebas y estudios de superficies alares es necesario debido a que la UNAH no posee los equipos adecuados para realizar un estudio teórico-práctico de los efectos aerodinámicos sobre diferentes formas y superficies.

Con este proyecto se podrán ver beneficiados todos los alumnos interesados en realizar prácticas de laboratorios y visualizar los cálculos matemáticos vistos en la clase teórica, pero la directa beneficiada será la Universidad; porque se podrá mejorar algunas investigaciones así mismo relacionar estudios realizados en diversos programas de simulación y comparar resultados de rendimiento mediante una base de datos.

El proceso investigativo definió los antecedentes, presentando así la problemática a analizar, con sus objetivos y fases del proceso de investigación.

Posteriormente se presentan los Resultados de la Investigación, brindando a través de esta la descripción técnica del Túnel Aerodinámico de la UNAH, los proveedores de dicho modelo, así como de sus accesorios y su funcionalidad desde la perspectiva pedagógica.

En las Conclusiones, es donde los investigadores brindan las consecuencias producto del estudio y del análisis de una serie de datos.

El artículo de esta investigación finaliza con los apartados de Bibliografía, que enuncia los documentos o referencias consultadas. 


\section{DEFINICIÓN DEL PROBLEMA}

La reciente creación del Departamento de Ciencias Aeronáuticas en la Facultad de Ciencias Espaciales de la Universidad Nacional Autónoma de Honduras ha generado la necesidad de carreras universitarias propias del campo, por lo que equipos, herramientas, simuladores o implementos didácticos para su enseñanza son necesario para la correcta educación en esta área.

Por lo que la problemática planteada fue:

La necesidad del acondicionamiento y el diseño de un túnel de viento acorde a las necesidades didácticas y pedagógicas para la enseñanza de materias de ciencias aeronáuticas propias de programas de estudio en la educación superior.

\section{DATOS Y METODOLOGÍA}

\section{Los Datos}

La presente investigación utilizo prevalentemente datos cualitativos, por esta razón se fundamentó en cualidades y características del túnel de viento de la UNAH, como objeto de estudio y, aunque no excluye el uso de datos cuantitativos, estos solo fueron utilizados para apoyar aspectos cualitativos.

Las variables utilizadas fueron:

- Variable dependiente: Características de diseño establecidas por el fabricante del túnel de viento que posee la UNAH.

- Variable independiente: Diseño y modificaciones propuestas por las tendencias de modernización aplicables al túnel de viento que posee la UNAH.

\section{Pruebas en Túneles de Viento (Ruíz, 2007)}

Los túneles aerodinámicos, también llamados túneles de viento (traducción literal de la expresión inglesa wind tunnel), son instrumentos científico-tecnológicos cuya aplicación es la generación de una corriente fluida de propiedades conocidas para la medida de las acciones del flujo sobre obstáculos de muy diversa naturaleza, aeronáuticos o no, y más generalmente para el estudio de los fenómenos físicos en los que el aire en movimiento juega un papel dominante, estudio de estelas, dispersión de contaminantes, apantallamientos, y otros. 
La finalidad de los ensayos en túnel aerodinámico es suministrar información sobre las particularidades del flujo en las proximidades del cuerpo en consideración, proveer información sobre la distribución de presiones y sobre las cargas globales producidas por la corriente en el obstáculo objeto de interés y, si el objeto del ensayo es flexible y susceptible de experimentar fenómenos aeroelásticos (la respuesta dinámica de las estructuras ante fuerzas aerodinámicas), así como proporcionar los datos necesarios sobre las vibraciones inducidas por el flujo.

El auge de la aeronáutica ha multiplicado extraordinariamente las necesidades de ensayos en túnel aerodinámico (véase la figura 1), habiéndose producido la misma situación en otros muchos campos de la ingeniería en los que también los túneles aerodinámicos constituyen una herramienta primaria para generar conocimiento $y$, aunque en mucha menor medida, en la biología.

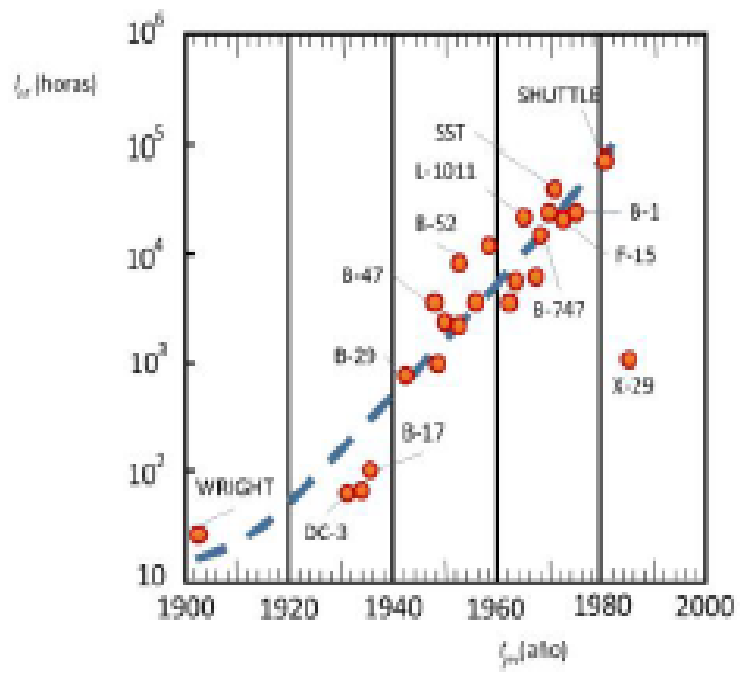

Figura 1. Tiempo de ensayos en túnel, empleado en el desarrollo de distintas aeronaves antes del Primer vuelo y la fecha del primer vuelo (Ruíz, 2007).

Cuando se ensayan modelos a escala, la validez de los resultados medidos requiere la existencia de semejanza geométrica, semejanza cinemática y dinámica entre el flujo alrededor del modelo y alrededor del obstáculo real, lo que en un amplísimo número de aplicaciones se traduce, aparte de que el modelo de ensayo sea una réplica geométrica del cuerpo real, en la igualdad de ciertos parámetros 
adimensionales en ambos flujos, el real y el generado en el túnel. De entre estos parámetros, los más relevantes en aeronáutica son sin duda el número de Mach y el número de Reynolds. El número de Mach, $\mathrm{M}=\mathrm{V} / \mathrm{Vs}$ es el cociente entre la velocidad de la corriente incidente $\mathrm{V}$ y la velocidad del sonido $\mathrm{Vs}$ en el medio que se mueve esta corriente. Este parámetro es de gran importancia en los flujos de elevada velocidad, donde las variaciones de densidad debidas a la presión dinámica son significativas, pero es irrelevante a bajas velocidades, por debajo de $400 \mathrm{~km} / \mathrm{h}$, por fijar un número, donde los efectos de la compresibilidad del fluido son apenas perceptibles. El número de Reynolds $(R e)$ es un número adimensional utilizado en mecánica de fluidos, diseño de reactores y fenómenos de transporte para caracterizar el movimiento de un fluido. En ingeniería aeronáutica el flujo sobre la capa límite de la corriente de aire es sumamente importante en donde la transición de flujo laminar a turbulento ocurre normalmente para valores de número de Reynolds entre medio millón y 10 millones.

\section{Tipos de túneles aerodinámicos}

Es así que se tiene en cuenta las diversas aplicaciones de los túneles aerodinámicos, entendiéndose que la mayoría de los mismos estén construidos para un cierto uso específico, lo que suele condicionar muchos aspectos del diseño. Se comprende que un túnel para aplicaciones de aerodinámica no aeronáutica, orientado hacia ensayos de edificios, puentes y otras estructuras de la ingeniería civil, que funciona en régimen incompresible, ha de tener unos requisitos muy distintos a los de un túnel supersónico; o que las necesidades de un ensayo de calibración de anemómetros difieran notablemente de las de los ensayos de medidas de cargas aerodinámicas sobre obstáculos no fuselados. Todo ello hace que exista una amplia variedad de túneles aerodinámicos, pudiéndose encontrar una gran diversidad.

Los túneles pueden ser subsónicos, transónicos, supersónicos o hipersónicos, variando entre ellos la forma y el tamaño de la cámara de ensayos (Figura 2). 


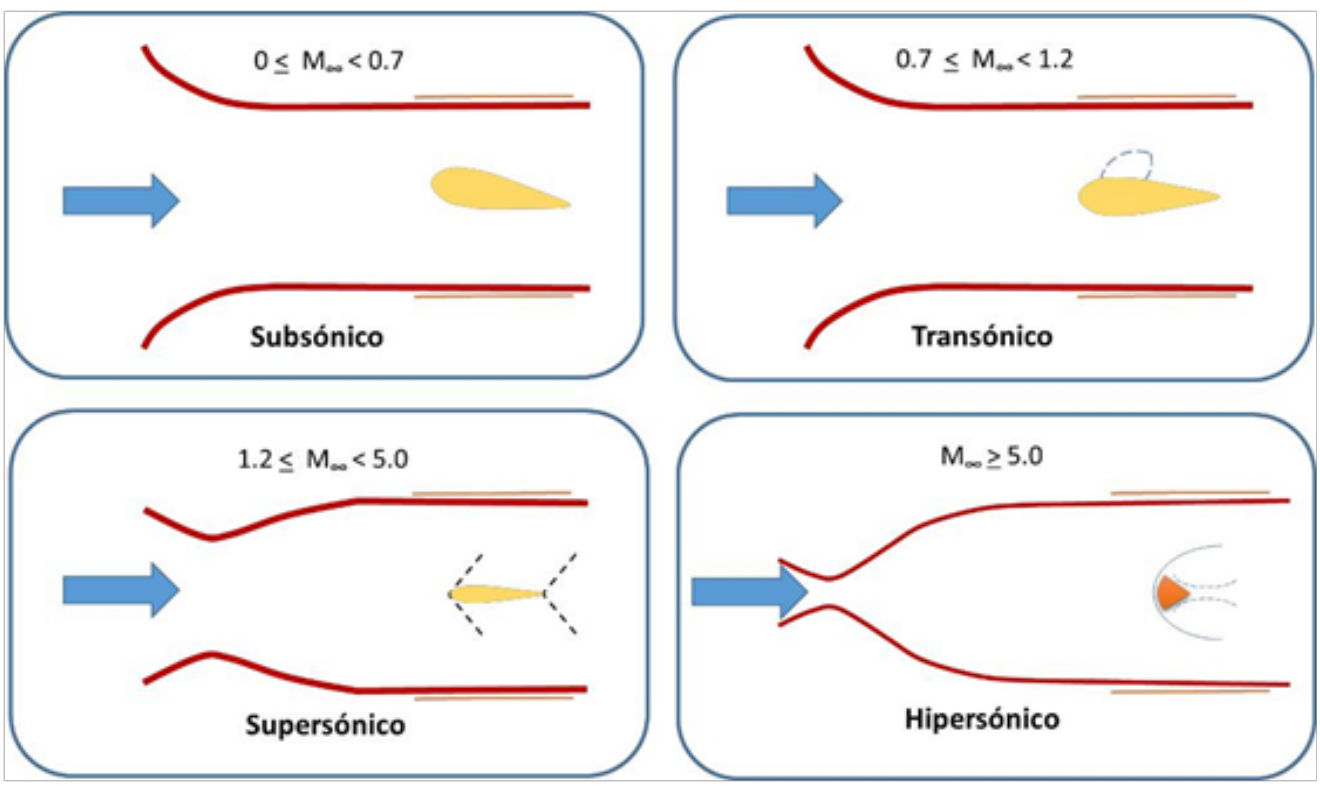

Figura 2. Variación de la forma de la cámara de ensayos de un túnel en función del número de Mach, Mœ (Ruíz, 2007).

En relación con la geometría global del conducto, los túneles aerodinámicos pueden ser, según tengan o no conducto de retorno, de circuito fluido cerrado, con un circuito de retorno específico, o circuito fluido abierto, donde el aire se toma directamente de la atmósfera y se descarga posteriormente a esta o bien retorna a través del local donde está el túnel.

Respecto a la cámara de ensayos, en los túneles subsónicos esta puede ser de sección abierta, es decir, sin paredes laterales, o cerrada (con paredes laterales). Aunque esta pareja de alternativas parece conducir a únicamente tres tipos de túnel (si la corriente retorna a través del local no es recomendable emplear una cámara de sección abierta por las interferencias entre la vena fluida de la cámara y el flujo de retorno), la realidad es que dentro de estos tres tipos básicos la variedad de diseños es enorme.

En la figura 3 se muestran de forma esquemática las plantas de los tres tipos básicos de túneles. En un túnel sin circuito de retorno el aire pasa a través del conducto, normalmente organizado en la secuencia tradicional de sección de entrada (U), contracción $(\mathrm{C})$, cámara de ensayos $(\mathrm{E})$, difusor de adaptación a la sección de 
ventiladores $(A)$, ventilador $(V)$ y difusor de salida (D). En los túneles con conducto de retorno $(R)$ este puede ser único, doble o incluso anular.

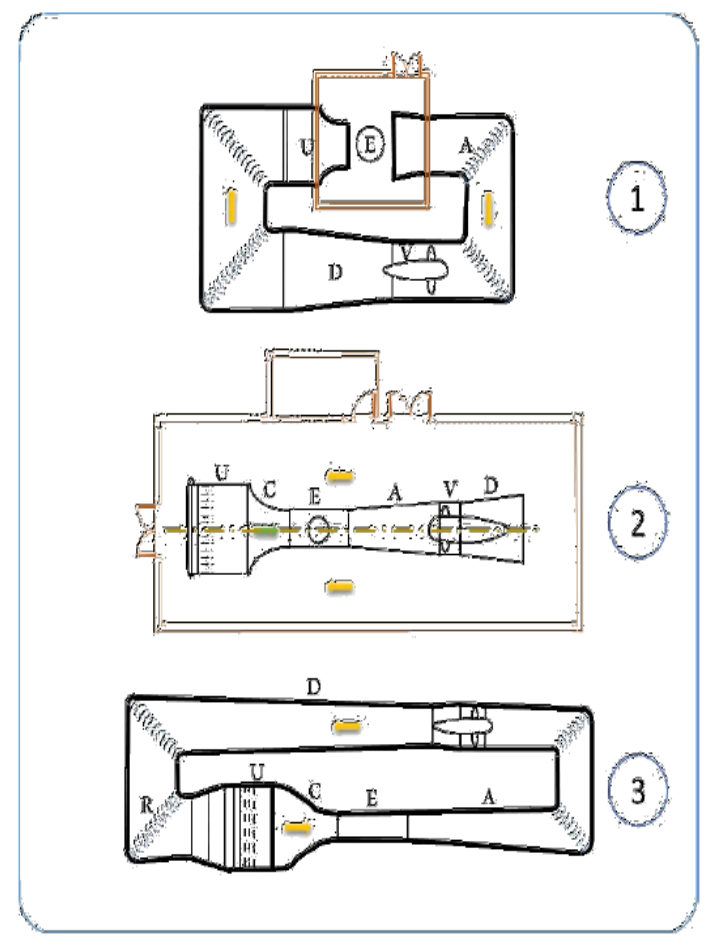

Figura 3. Tipos básicos de túnel aerodinámico: de sección de ensayos abierta y con circuito de retorno (1), de sección de ensayos cerrada y sin circuito de retorno (2), y de sección de ensayos cerrada y con circuito de retorno (3). Las letras identifican los distintos componentes definidos

(Ruíz, 2007).

Cada configuración presenta sus ventajas y sus inconvenientes. Así, en los túneles sin circuito de retorno, el coste de fabricación suele ser menor, pero también lo es el rendimiento energético de la instalación, además estos túneles son más ruidosos. En los abiertos a la atmósfera su operación puede depender de condicionantes meteorológicos.

Las ventajas de los túneles con circuito de retorno específico es que ofrecen mayores posibilidades de control del flujo inducido, disminuyendo el consumo de energía así como del ruido, pero por el contrario la inversión económica inicial es más alta. 


\section{El Túnel de Viento Educativo}

El Túnel de viento educativo es un equipo sencillo y seguro de operar. Se ofrece a centros de educación e investigación como instalación autónoma completa. equipo principal consta del túnel con un sistema compensador de dos componentes y un indicador de la velocidad en el aire.

El aire entra en la sección de prueba a través de una contracción cuidadosamente diseñada, seguida de un enderezador de flujo tipo panal diseñado para asegurar que el flujo sea constante tanto en magnitud como en dirección, y tenga un perfil de velocidad transversal plano. Un difusor de ángulo pequeño en el extremo de salida contribuye a la estabilidad del flujo en la sección de prueba. Un ventilador está ubicado en la salida de la sección difusora. El ventilador es impulsado por un motor alimentado por corriente alterna que es controlado por una unidad inversora de control de velocidad, lo que permite el control uniforme de la velocidad en el aire.

La sección de prueba paralela cilíndrica está fabricada en material acrílico transparente y puede ser retraído sobre raíles para permitir un acceso sin obstrucciones a los modelos.

El compensador de dos componentes consta de un par de compensadores soportados sobre filos en ejes perpendiculares entre sí y paralelos y perpendiculares al centro axial del túnel. Los componentes de sustentación y resistencia de la fuerza ejercida sobre los modelos bajo prueba son compensados deslizando pesos por los brazos del compensador hasta obtener un estado de deflexión nula.

Las graduaciones, en unidades de fuerza, permiten la lectura directa de la sustentación y la resistencia. El conjunto completo está conectado a un sencillo amortiguador de aceite.

La precisión del túnel y su instrumentación también hacen que sea adecuado para trabajos de estudiantes universitarios y proyectos de investigación sencillos.

El estándar de un túnel de viento educativo hace posible una amplia gama de mediciones y demostraciones. He aquí una selección usando los modelos y la instrumentación que el modelo que posee la UNAH podría realizar (Armfield, 2015):

1. Investigación del desarrollo de la capa límite en una placa plana mediante la medición de la distribución de carga total.

2. Estudios de visualización del flujo alrededor de una sección aerodinámica. 
3. Medición de la distribución de la presión alrededor de una sección aerodinámica a diferentes ángulos de ataque.

4. Medición de la distribución de la presión alrededor de un cilindro

5. Medición de sustentación y resistencia en una sección aerodinámica con ranura en el borde de ataque y flap en el borde de escape.

6. Mediciones de velocidad y distribución de la presión usando un tubo de Pitot estático y sonda de guiñada.

7. Medición de resistencia para una selección de modelos de diferentes formas pero con diámetro ecuatorial común.

8. Demostración de la vibración aeroelásticos de una sección aerodinámica.

9. Calibración del indicador de velocidad del túnel de viento usando un tubo de Pitot estático y un manómetro inclinado.

10. Investigación de la estela dejada por un cilindro o una sección aerodinámica usando un rastrillo de medición de estela.

\section{LAMETODOLOGÍA}

La metodología diseñada para esta investigación se basó básicamente en el análisis del túnel de viento que se encuentra en la UNAH, bibliografía consultada y la realización de consultas técnicas a fabricantes.

Los pasos se describen a continuación:

\section{Análisis Consulta bibliográfica.}

Las lecturas leídas de manera preliminar fueron de gran ayuda para definir el campo conceptual en el cual se propuso la investigación, en ese sentido se consultaron una serie de documentos referentes al uso y diseño de un túnel de viento por fabricantes y expertos.

\section{Construcción de marco referencial}

Con las lecturas que se consultaron se establecieron los conceptos principales que se debían conocer para generar un análisis basado en bibliografía especializada 
en aerodinámica y sus ensayos en ambientes controlados como el túnel de viento, por tal razón se consultaron:

Libro Aeronáutica Moderna escrito por Curtis E. LeMay (LeMay, 1965); De este documento se tomaron los conceptos básicos de la aerodinámica aplicada, nomenclatura y definiciones utilizadas en el diseño y operación de aeronaves modernas, el ambiente en que se desenvuelven y los efectos que este produce sobre ellas. Revisión de la información científica ofrecida a través del libro Theoretical Aerodynamics (Milne-Thomson, 1973); Se toma como fuente esencial para el establecimiento teórico de las pruebas matemáticas al comportamiento de los fluidos sobre las superficies aerodinámicas, las que pueden ser comprobadas a través de pruebas de laboratorio, haciendo uso de un túnel de viento.

Definir las Características Aerodinámicas de los Perfiles de Ala a través de la revisión del Libro Aerodinámica del Vuelo: Aves y Aeronaves (Ruíz, 2007); Se establecerán las características aerodinámicas de los perfiles de ala para su aplicación a los modelos a ser utilizados en las prácticas de laboratorio aerodinámico.

Desarrollar proyectos de laboratorio utilizando como referencia el documento Operating Instructions for Aerolab (Aerolab, 1991); Se examinó una serie de actividades de laboratorio mediante las cuales se pueden llevar a la práctica muchos de los principios teóricos de la aerodinámica con el uso de un túnel de viento con las características del que ya posee la UNAH en el Laboratorio de Máquinas Herramientas, de la Facultad de Ingeniería.

\section{Recolección de información de campo}

Tomando como referencia fundamental al marco teórico se consultó al Fabricante del Túnel de Viento AEROLAB y al fabricante coreano Eunha Fluid Science, sobre los suministros de los accesorios y las características técnicas de estos para las pruebas del laboratorio aerodinámico, sus costos y forma de adquisición.

\section{RESULTADOS}

\section{Análisis de los datos obtenidos.}

Una vez que se hicieron las consultas vía correo electrónico la transcripción de los aportes fueron redactados procurando al máximo posible reflejar fielmente lo propuesto por las empresas consultadas. En el caso de los textos consultados se hicieron las citaciones de los párrafos extraídos directamente de los documentos revisados. 
Discusión de los datos obtenidos.

En esta parte se seleccionó datos que cumplieron los dos requisitos: que fueron acordes con la metodología propuesta y aportaron datos útiles para proponer alternativas de solución al problema propuesto. En tal sentido, se hizo un análisis de tipo cuantitativo y cualitativo y se tomó nota de las ideas recurrentes en las empresas consultadas haciendo una comparación con la información brindada en los textos consultados y la carrera propuesta por el Departamento de Ciencias Aeronáuticas de la UNAH.

Presentación de conclusiones del estudio.

Una vez delineados los resultados de la investigación se preparó el presente informe a ser entregado y discutido en el Departamento de Ciencias Aeronáuticas, con el objetivo de ser preparado para ser publicado en la Revista Científica "Ciencias Espaciales". Para fines de divulgación se preparará y hará una presentación destinada a los profesionales e instituciones que ofrecieron información.

\section{Características técnicas del Túnel de Viento Educativo existente en la UNAH}

El primer paso para la propuesta de restauración del túnel de viento fue evaluar las partes existentes proporcionadas por la Universidad. Esto sirve de base para dimensionar secciones faltantes así como para elaborar un diseño didáctico, que permitirá a los alumnos observar y cualificar los fenómenos del aire en movimiento. Este diseño didáctico, permite además modificar y/o agregar componentes, modificando así los resultados esperados de las pruebas de laboratorio que a futuro se realizarán en este túnel de viento. La imagen del túnel de viento cuando fue adquirido por la UNAH es muy similar a la figura 4.

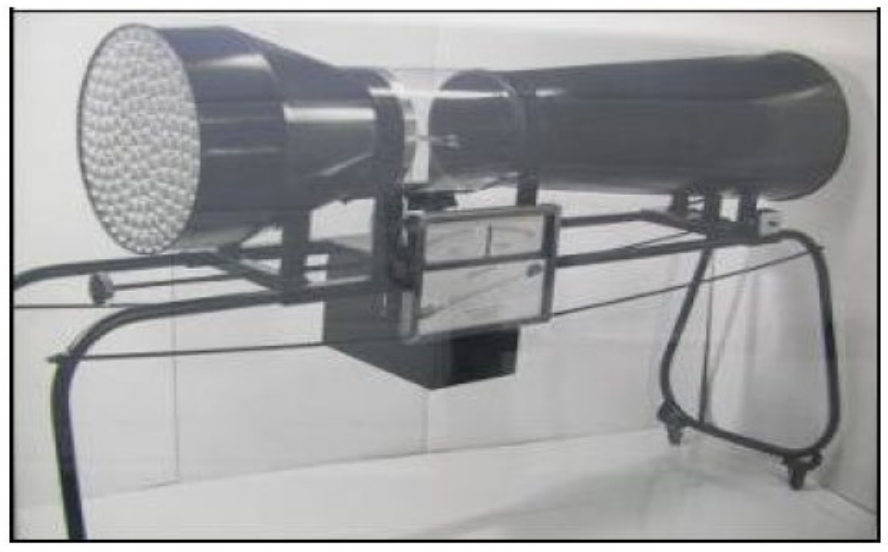

Figura 4. Modelo Publicitario de AEROLAB. 
El equipo propuesto a condicionar debe contar con lo mínimo con las siguientes partes: (ver figura 5).

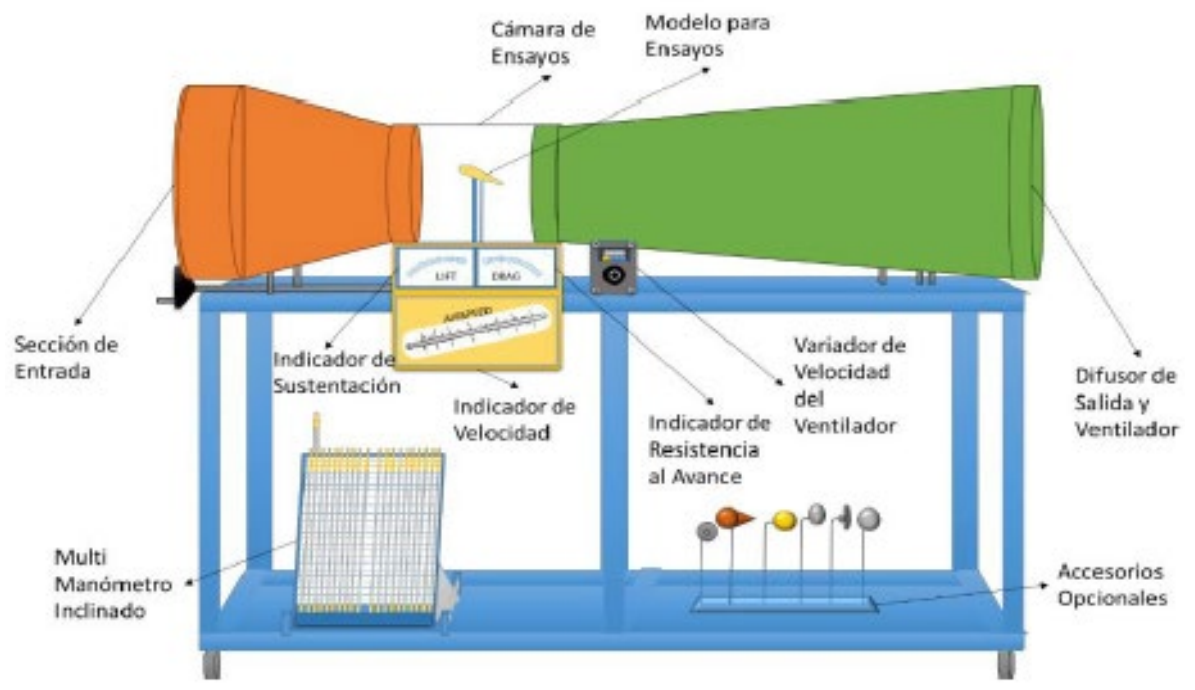

Figura 5. Esquema del Túnel de Viento original del modelo que posee la UNAH.

\section{Sección de Entrada}

\section{Cámara de Ensayos}

3. Difusor de Salida y Ventilador

4. Indicador de Velocidad

5. Indicador de Sustentación

6. Indicador de Resistencia al Avance

7. Variador de Velocidad del Ventilador

8. Multi manómetro Inclinado

9. Accesorios Opcionales 
En el Túnel de Viento que posee la UNAH, se pudieron identificar partes que todavía están presentes y utilizables, luego de ser habilitadas, las cuales se ven en la figura 6.

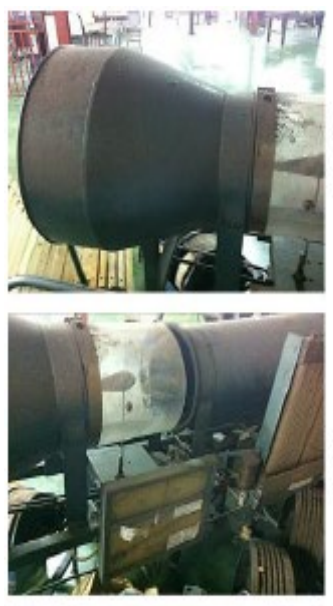

Sección de Entrada

Cámara de Ensayos

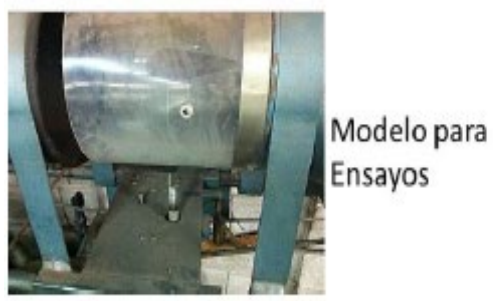

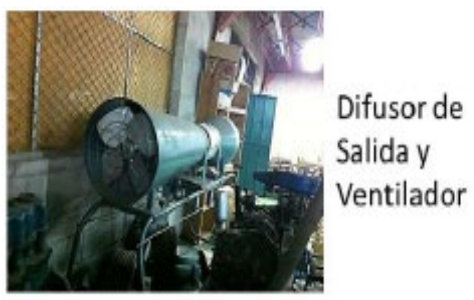

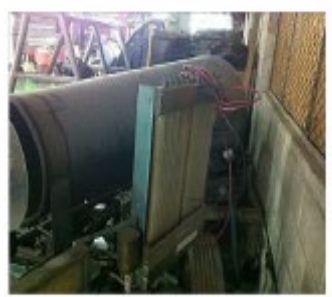

Multi

Manómetro

Inclinado

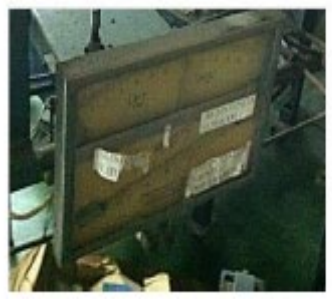

Indicador de Sustentación Indicador de Velocidad

Indicador de Resistencia al Avance

Figura 6. Partes identificadas como presentes en el Túnel de Viento que posee la UNAH.

Las piezas como el Variador para la Velocidad del Ventilador y Modelos de Ensayo (a diferencia de la bola con extensión) no se pudieron encontrar, por lo que es necesaria su fabricación o su compra, para poder completar el equipo de simulación y pruebas. 
En resumen, el estatus técnico del túnel de viento de la UNAH puede visualizarse en la Tabla No.1:

Tabla 1. Resumen del Estatus Técnico del Túnel de Viento de la UNAH

\begin{tabular}{|c|c|c|c|}
\hline Accesorio & Componentes & Caracteristicas & $\begin{array}{c}\text { Estatus en el Tunel de Viento } \\
\text { de la UNAH }\end{array}$ \\
\hline \multirow[t]{3}{*}{ Ventilador } & Velocidad del Aire & 20 metros por segundo (Máximo) & \multirow[t]{3}{*}{ Funcional } \\
\hline & Potencia del Motor & $1 / 2 \mathrm{Hp}$ & \\
\hline & Fuente de Electricidad & 110 voltios $(60 \mathrm{Hertz})$ & \\
\hline \multirow[t]{3}{*}{ Túnel de Viento } & Sección de Prueba & $30 \mathrm{~cm}$ de Diámetro por $35 \mathrm{~cm}$ de largo & \multirow[t]{3}{*}{ Funcional } \\
\hline & Dimensión Total & $\begin{array}{l}70 \mathrm{~cm} \text { de Ancho, } 200 \mathrm{~cm} \text { de Largo y } 167 \mathrm{~cm} \text { de } \\
\text { Alto }\end{array}$ & \\
\hline & Peso & 303 libras & \\
\hline \multirow{6}{*}{$\begin{array}{l}\text { Accesorios } \\
\text { Estándar }\end{array}$} & Tubo de pitot estático & $3 \mathrm{~mm}$ de Diámetro y $30 \mathrm{~cm}$ de largo & No se encontró \\
\hline & \multirow[t]{5}{*}{ Multi Manómetro Inclinado } & Distancia de Lectura desde 0 a $30 \mathrm{~cm}$ & \multirow[t]{5}{*}{ Funcional } \\
\hline & & Numero de tubos son 20 & \\
\hline & & Inclinación desde $20^{\circ}$ a $90^{\circ}$ & \\
\hline & & Reservorio de nivel variable & \\
\hline & & Colector de metal con 20 conexiones & \\
\hline \multirow{6}{*}{$\begin{array}{l}\text { Accesorios } \\
\text { Opcionales }\end{array}$} & & orma Aerodinámica Alar & No se encontró \\
\hline & \multicolumn{2}{|c|}{ Ala para medir presión en diferentes áreas } & No se encontró \\
\hline & \multicolumn{2}{|c|}{ Modelos para pruebas de Resistencia al Avance } & No se encontró \\
\hline & \multicolumn{2}{|c|}{ Superficie para pruebas de capa limite } & No se encontró \\
\hline & \multicolumn{2}{|c|}{ Simulación de superficies secundarias Slot y Flaps } & No se encontró \\
\hline & \multicolumn{2}{|c|}{ Equipo de rastrillo de humo } & No se encontró \\
\hline
\end{tabular}

Fuente: elaboración propia en base a observación.

\section{Proveedores de Túneles de Viento y Accesorios}

Los proveedores, específicamente para el modelo que la UNAH posee, solo son dos, de acuerdo a los encontrados durante esta investigación y son:

- La empresa estadunidense AEROLAB, que tiene la página web www.aerolab. com

- La empresa coreana Eunha Fluid Science, que tiene la página web http://www. ehf.co.krl

Aunque la primera fue el fabricante original de este modelo, ya no tiene el stock de repuestos para el modelo que la UNAH posee, pero las modificaciones a las piezas de túneles de viento más modernos que ellos fabrican no tienen tantas variantes, por lo que su adaptación es factible. 
En el caso del segundo, el que incluso lo sigue fabricando con el número de producto EH-EWT-2500, cuenta con todo el stock de repuestos, siendo una gran oportunidad para la restauración del modelo de la UNAH. Los costos sugeridos por dichas empresas son los siguientes:

Tabla 2. Costos sugeridos de los accesorios faltantes por las empresas consultadas

\begin{tabular}{|c|c|c|c|}
\hline Descripción & $\begin{array}{l}\text { Modelo según Empresa } \\
\text { Eunha Fluid Science * }\end{array}$ & $\begin{array}{l}\text { Precio Empresa } \\
\text { AEROLAB }\end{array}$ & $\begin{array}{l}\text { Precio Empresa Eunha } \\
\text { Fluid Science }\end{array}$ \\
\hline Tubo de pitot estático & Sin Número & $\begin{array}{ll} & 270.00\end{array}$ & $\$ 100.00$ \\
\hline Forma Aerodinámica Alar & Modelo EWT-1 & No Cotizado & $\$ \quad 1,200.00$ \\
\hline Ala para medir presión en diferentes áreas & Modelo EWT-2 & \$ $1,320.00$ & $\$ 1,100.00$ \\
\hline $\begin{array}{l}\text { Modelos para pruebas de Resistencia al Avance (5 } \\
\text { piezas) }\end{array}$ & Modelo EWT-3 & $\begin{array}{l}S^{\prime} \\
780.00\end{array}$ & $\$ 900.00$ \\
\hline Superficie para pruebas de capa limite & Modelo EWT-4 & 880.00 & No Cotizado \\
\hline Simulación de superficies secundarias Slot y Flaps & Modelo EWT-5 & 970.00 & No Cotizado \\
\hline Equipo de rastrillo de humo & Modelo EWT-6 & 340.00 & No Cotizado \\
\hline
\end{tabular}

* La empresa AEROLAB no mostro códigos para sus modelos.

\section{DISCUSIÓN}

Un túnel de viento utilizado en la enseñanza pretende ilustrar de manera didáctica los efectos del aire sobre objetos, permitiendo la realización de investigaciones, diseños, prácticas de laboratorio para estudiantes, proyectos de tesis, trabajos en conjunto con la industria y muchos más, abriendo la posibilidad de generar un pequeño centro de investigación, y enriquecer las actividades académicas que allí se desarrollen.

Al agregar el túnel de viento al laboratorio de aerodinámica, se da un gran paso para ampliar su rango de servicios. El túnel de viento servirá como herramienta en la enseñanza y para la experimentación e investigación de modelos aerodinámicos y otras máquinas que interactúan con el aire en movimiento, haciendo uso de un manual de prácticas de laboratorios, que se desarrollara al momento que el túnel de viento de la UNAH este funcional.

En la UNAH es necesario desarrollar este proyecto de reacondicionamiento del túnel de viento ya existente, pues en el medio no se encuentran posibilidades de realizar pruebas y mediciones aerodinámicas ni de mecánica de fluidos. La Universidad cuenta con la infraestructura y respaldo para sostener e impulsar este 
servicio, que estaría a disposición de estudiantes, profesores y eventualmente a disposición de la industria.

A futuro se podría obtener uno más moderno como se puede apreciar en la figura 7 que muestra el Túnel de Viento que en la actualidad fabrica la empresa AEROLAB, el cual es utilizado en la enseñanza de la aerodinámica, haciendo uso de sistemas computacionales, y accesorios más ergonómicos.

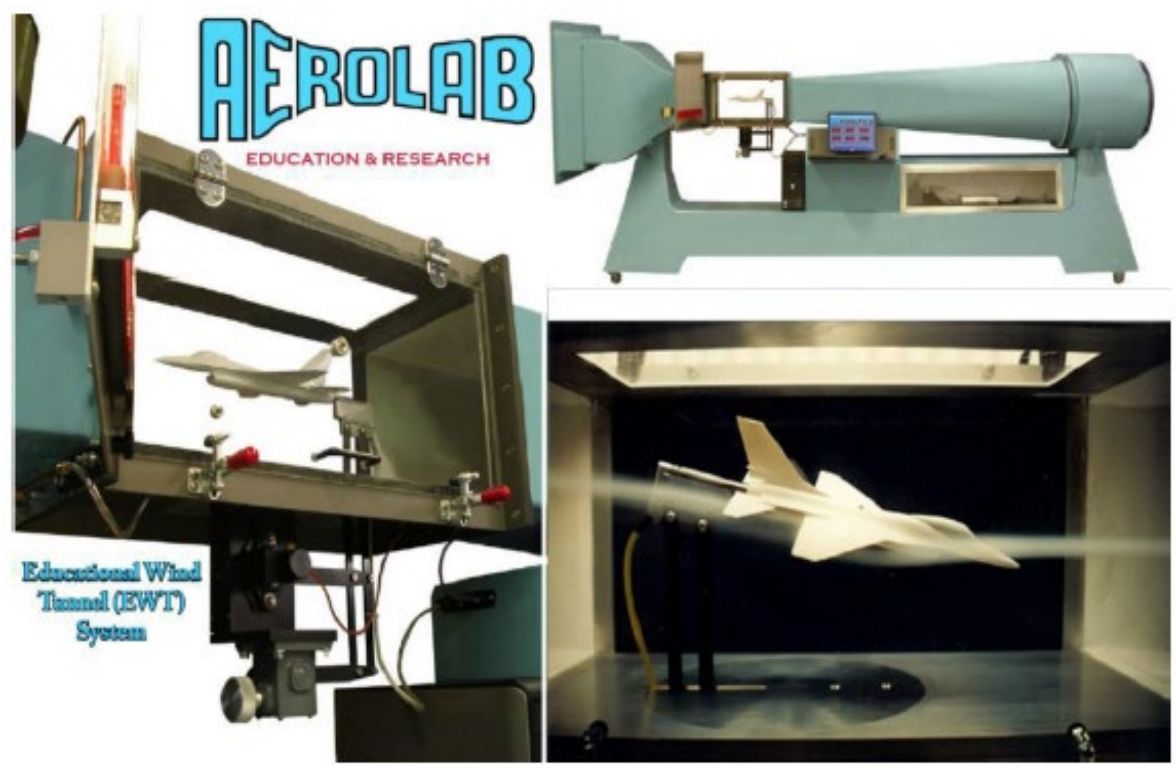

Figura 7. Túnel de Viento Moderno para enseñanza sobre aerodinámica (AEROLAB, 2015) 


\section{CONCLUSIONES}

1. El proyecto ofrece en conjunto con el soporte institucional, la posibilidad de realizar trabajos que combinen los aspectos teóricos y prácticos, brindando apoyo a la labor docente, investigativa y de la industria en el campo de la aerodinámica y mecánica de fluidos.

2. Las características del túnel de viento cubren las necesidades propuestas de simulación y prácticas de laboratorio expuestas en el espacio de aprendizaje denominado "Aerodinámica y Mecánica de Vuelo" desarrollado por el Departamento de Ciencias Aeronáuticas.

3. A pesar de los avances de simulación gráfica basados en sistemas computacionales para el análisis del comportamiento de superficies alares y otro tipo de formas, aún es necesario emplear métodos experimentales, en los cuales el túnel de viento es una excelente herramienta, además el conocimiento de las experimentaciones físicas, facilitará el manejo y aplicación de los modelos computarizados que se pueden encontrar por medios virtuales.

4. Para optimizar su funcionamiento en un ambiente didáctico, el túnel de viento tal y como se encuentra en el laboratorio de Máquinas Herramientas de la Carrera de Ingeniería Mecánica de la UNAH, debe ser modificado con los accesorios fabricados por las empresas ofertantes disponibles a nivel internacional; la presente investigación ofrece cotizaciones de dos de estas empresas..

5. Por lo que respecta a los aspectos físicos del túnel de viento, su diseño de sistema de adquisición de datos debe ser mejorado y reacondicionado, visualizando a futuro el desarrollo e implementación de un sistema automatizado.

6. Por lo que respecta al ambiente en el que funcionará el túnel de viento, el recinto donde será instalado, debe contar con la capacidad de tomar aire fácilmente y expulsarlo de forma libre, preferiblemente hacia afuera del recinto y sin obstrucciones de ningún tipo, evitando de esta manera la turbulencia y contaminación del sistema.

7. La línea de investigación en el campo de la aerodinámica no existe en Honduras, el tener a disposición un túnel de viento operativo en la UNAH dará oportunidad al País a incursionar en este tipo de investigaciones, estableciendo con esto un espacio desde el cual se pueda hacer aportaciones científicas en una dinámica interdisciplinaria. 
8. Un tema pendiente que se deriva de la presente investigación es el diseño de un manual de prácticas de laboratorio que abarquen el uso del Túnel de Viento de la UNAH una vez que esté operativo, generará una manera medible y operativa para que el alumno pueda visualizar lo aprendido en clases de manera práctica.

\section{AGRADECIMIENTOS}

A la Coordinación de la Carrera de Ingeniería Mecánica, específicamente al Laboratorio de Maquinas Herramientas por su apoyo al facilitarnos el acceso al Túnel de Viento, ya que esto proporciono información relevante para el desarrollo de la investigación.

A las empresas diseñadoras de los túneles de viento internacionales consultadas, ya que sin su apoyo no se hubiera podido establecer las características técnicas de los túneles de viento analizados.

Al Licenciado Alex Matamoros, del Departamento de Ciencias Aeronáuticas de la UNAH por su valiosos aportes en el diseño y redacción de este artículo.

\section{BIBLIOGRAFÍA}

- AEROLAB. (6 de Noviembre de 2015). About. Obtenido de History: http://www. aerolab.com/about/

- AEROLAB. (22 de Octubre de 2015). AEROLAB. Obtenido de Acerca de AEROLAB: http://www.aerolab.com/about/

- AEROLAB. (27 de Noviembre de 2015). Educational Wind Tunnel (EWT) System. Obtenido de www.aerolab.com: www.aerolab.com

- Armfield. (24 de Noviembre de 2015). Túnel de Viento Subsónico. Obtenido de MECÁNICA DE FLUIDOS APLICADA: http://discoverarmfield.com/

- FAA, F. A. (2009). Pilot's Handbook of Aeronautical Knowledge. Washington: Government Printing Office. 
- Kim, S. (06 de Noviembre de 2015). The Business Montly. Obtenido de Wind Tunnel Maker Blowing Strong After Six Decades: http://www.bizmonthly.com/ wind-tunnel-maker-blowing-strong-after-six-decades/

- NASA. (2015). NASA. Obtenido de Wind Tunnels of NASA: http://www.hq.nasa. gov/office/pao/History/SP-440/contents.htm

- Ruíz, J. M. (2007). Aerodinámica del Vuelo: Aves y Aeronaves. Madrid: Centro de documentación y Publicaciones de AENA.

- Sampieri, R. H. (2010). Metodología de la Investigación 5ta Edición. Mexico: Mc Graw Hill.

- Sickle, N. D. (1985). AERONAUTICA MODERNA. AVIACION BASICA APLICADA. Washington:

- EDICIONES PARANINFO.

- UNAH. (1994). Ley de Educación Superior de Honduras. Tegucigalpa: Universidad Nacional Autónoma de Honduras. 